Société d'histoire de la révolution de 1848 et des

révolutions du XIXe siècle

19 | 1999

Aspects de la production culturelle au XIXe siècle

\title{
Loire et Rhône
}

\section{OpenEdition}

Journals

Édition électronique

URL : http://journals.openedition.org/rh19/192

DOI : $10.4000 /$ rh 19.192

ISSN : $1777-5329$

\section{Éditeur}

La Société de 1848

\section{Édition imprimée}

Date de publication : 1 décembre 1999

Pagination : 176-179

ISSN : 1265-1354

Référence électronique

" Loire et Rhône », Revue d'histoire du XIXe siècle [En ligne], 19 | 1999, mis en ligne le 26 août 2008, consulté le 15 septembre 2020. URL : http://journals.openedition.org/rh19/192

Ce document a été généré automatiquement le 15 septembre 2020

Tous droits réservés 


\section{Loire et Rhône}

\section{CORRESPONDANTS}

Claude Latta - Loire et Rhône

Colloques et numéros spéciaux de revues

* Le 150e anniversaire de 1848 : numéro spécial des Cahiers d'histoire et colloque La revue Cahiers d'histoire a publié un intéressant numéro spécial, 1848 en provinces [JeanLuc Mayaud, dir.] dont la Revue d'histoire du XIXe siècle a rendu compte dans son $n^{\circ} 18$. L'Université Lyon-2 (Jean-Luc Mayaud) et l'Institut d'études politiques de Lyon (Paul Bacot) ont, d'autre part, organisé le colloque 1848 à Lyon et dans sa région: usages, discours, images (27 et 28 novembre 1998) qui s'est articulé autour de quatre thèmes : " Le choc de 1848 ", " 1848 dans les urnes ", " 1848 en images et en chansons " et " Les mémoires de 1848 ". Souhaitons que les Actes en soient rapidement publiés.

\section{* Colloque Benoît Malon, 24 et 25 avril 1999}

Le colloque organisé par l'Association des amis de Benoît Malon et par l'Université de Saint-Étienne a eu lieu, en présence d'un auditoire nombreux, les 24 et 25 avril 1999 à Montbrison et à Précieux (Loire), le village natal de Benoît Malon. Il était placé sous la présidence de Marc Vuilleumier, de l'Université de Genève. Les Actes seront publiés fin 1999 par le Centre d'études foréziennes. Les communications du colloque étaient groupées autour des thèmes suivants : l'homme et l'histoire d'un révolutionnaire (les souvenirs d'enfance, l'influence de sa compagne Léodile Champseix, alias la romancière André Léo, l'exil en Suisse et en Italie), l'action politique (le Congrès socialiste de SaintÉtienne en 1882), l'œuvre (le théoricien du socialisme, la pensée économique, l'historien de la Commune, l'auteur d'un roman historique), les influences et les amitiés (Jules Vallès, Alexandre Millerand, le socialiste dijonnais Eugène Marpaux).

Renseignements, adhésions, bulletins de souscription aux actes du colloque: Association des amis de Benoît Malon, mairie, 42600 Précieux.

* Colloque " Enseigner l'État " (IUFM de Lyon) 
L'IREHG (Institut de recherche sur l'enseignement de l'histoire et de la géographie), pôle de recherche de l'IUFM de Lyon et son directeur, Didier Nourrisson, organisent les 20, 21 et 22 janvier 2000, un colloque " Enseigner l'État ", à la charnière de la recherche et de la réflexion pédagogique. Les communications et les discussions en ateliers s'organisent autour de trois thèmes : État et pouvoir, l'État et ses valeurs, États et puissances. Publication des Actes en 2001 par la revue IREHG.

Renseignements : Didier Nourrisson, IUFM, Centre local de Saint-Étienne, 90 rue de la Richelandière, 42100 Saint-Étienne cedex.

Thèses, mémoires de maîtrise, publications

* Soutenance de thèse de Pascal Chambon (Université de Saint-Étienne)

Pascal Chambon, professeur d'histoire au collège Victor-de-Laprade de Montbrison, a soutenu, le 22 janvier 1999, sa thèse, consacrée à l'étude du département de la Loire sous le Premier Empire et, en particulier, aux problèmes de la guerre, de la conscription et de la résistance à cette conscription. Le jury était composé par Yves-Marie Bercé, président et Jean-Paul Bertaud, Serge Dontenwill, Jacqueline Bayon. La présentation de thèse de Pascal Chambon a été publiée: "Du Consulat à la Seconde Restauration: l'exemple d'une société provinciale entre guerre et paix, le département de la Loire ", dans Annales historiques de la Révolution française, $n^{\circ} 316,1999$, pp. 365-371.

* Des mémoires de maîtrise

Sous la direction de Didier Nourrisson, ont été soutenus en 1999 à l'Université de SaintÉtienne, une série de mémoires de maîtrise consacrés au XIXe siècle. Citons : Cyrille Pautrat, La résistance au coup d'État du 2 décembre 1851 à Saint-Étienne; Maxim Makou, Le 14 juillet sous la IIIe République à Saint-Étienne; Blandine Guichard, La vie religieuse dans la paroisse de Saint-Genest-Malifaux, 1801-1905.

* Publications de l'Université de Saint-Étienne

Nathalie Verney-Carron, Le ruban et l'acier, Saint-Étienne, 1999, $150 \mathrm{~F}$. Cette remarquable étude, parue en septembre 1999, brosse le tableau d'un secteur industriel - la rubanerie et l'armurerie - et de tout un groupe social, celui du patronat stéphanois au XIXe siècle.

* Une revue stéphanoise: Histoire et mémoire

L'Association des amis du vieux Saint-Étienne qui gère le musée historique de SaintÉtienne (Hôtel de Villeneuve, 13 bis rue Gambetta, Saint-Étienne) publie un bulletin qui vient de se transformer en une véritable revue, Histoire et mémoire. Signalons en particulier le numéro spécial ( $n^{\circ} 188$, octobre 1997) consacré à l'histoire des femmes qui regroupe, autour de deux grands thèmes, d'excellents articles, souvent issus de mémoires de maîtrise. Citons en particulier des études sur les femmes en prison à la maison d'arrêt de Saint-Étienne (Béatrice Majewski), l'avortement à Saint-Étienne (Armelle Patural) et les femmes dans les ateliers de rubanerie (Brigitte Reynaud). Roger Bellet, aujourd'hui disparu, a donné là l'un de ses derniers articles (" Une grande journaliste, Séverine ") et Madeleine Bosko-Primet évoque "Flora Tristan à SaintÉtienne ". D'autres numéros à thème sont parus : parmi eux, l'un est consacré à l'art du métal : nous sommes dans la ville des armuriers ( $n^{\circ} 195$, juillet 1999). 
Commémoration et publication

Le souvenir de la révolution de 1848 à Montbrison

En décembre 1998, une conférence de Claude Latta a évoqué, au Centre social de Montbrison, La révolution de 1848 et la Seconde République dans le Montbrisonnais. D'autre part, un comité s'est formé et a lancé une souscription pour la rénovation du monument des républicains qui, au cimetière de la ville, rappelle le souvenir de deux quarantehuitards foréziens, Martin Bernard et Eugène Baune. Ceux-ci sont aussi évoqués dans l'article de Claude Latta, " Martin Bernard, Eugène Baune et le combat républicain ", entretien avec Jean-Claude Vimont, publié par Trames, revue de l'IUFM de Rouen, $\mathrm{n}^{\circ} 5$, juin 1999, nº spécial consacré à République et républicains.

\section{In Memoriam}

\section{À la mémoire de Fernand Rude}

Le 19 septembre 1999, une plaque à la mémoire de Fernand Rude (1910-1990) ${ }^{1}$, a été apposée et inaugurée sur la façade du 29 du quai Saint-Vincent où il a vécu. Fernand Rude, historien des canuts lyonnais et du mouvement social, est l'un des présidents d'honneur de la Société d'histoire de la révolution de 1848, collaborateur du Bulletin et participant à plusieurs de nos colloques. Max Bobichon, son ami, et François Rude, son fils, rédacteur en chef de la revue Icare, membre de la Société d'histoire de 1848, prirent la parole pour évoquer l'homme, l'historien et le résistant.

\section{René Berchoud (1931-1999)}

Notre ami René Berchoud, membre de la Société d'histoire de la révolution de 1848, nous a quittés le 21 Juin 1999. Professeur de lettres à Besançon, puis à Bagnols-sur-Cèze et enfin à Feurs (Loire), il était à la fois un enseignant à l'extraordinaire rayonnement, un militant d'éducation populaire et un historien.

René Berchoud était d'abord passionné de littérature et d'histoire, savait faire partager cette passion à ses élèves. Connaissant tout Hugo, il avait donné une étude sur Inès de Castro, personnage qu'il aimait et qu'il sentait au cœur du mystère hugolien. Il s'intéressa aussi au "patois" (le franco-provençal) et, stimulé par les conseils de Marguerite Gonon, consacra un mémoire de DEA au patois de Néronde, soutenu à l'Institut Pierre-Gardette (Lyon). Le Bicentenaire de 1989 fut aussi, pour lui, l'occasion d'étudier la Révolution à Néronde et dans les monts du Lyonnais.

Mais, avant tout, René Berchoud croyait que l'on peut " changer la vie ", en particulier par l'éducation populaire et par la culture mise à la disposition de tous. Avec son épouse Micheline, il anima à Besançon le CCPPO (Centre culturel populaire de Palenteles-Orchamps) dont l'action, menée dans le climat de l'affaire Lip, a laissé un souvenir mythique. Henri Guillemin, Chris Marker, Patrice Chéreau, entre autres, furent alors au rendez-vous de cette extraordinaire aventure intellectuelle, menée par les habitants eux-mêmes. Passionné de théâtre, auteur à ses heures, René Berchoud a créé à Bagnolssur-Cèze le groupe théâtral "ça ira ". À Néronde, enfin, il fut à l'origine de l'ADN (Association des amis de Néronde) et de la revue Jarnicoton qui s'intéresse à l'histoire locale, au patois et à l'animation de son village. Ses écrits avaient du style, le sens de la formule : à le lire, on entend sa voix. Et il y avait l'homme, chercheur d'absolu, refusant les conventions et les faux-semblants, privilégiant les qualités du cœur, sensible à l'amitié, généreux de son temps et de son admiration, capable d'aider les autres, de les 
pousser à donner le meilleur d'eux-mêmes.

René Berchoud était d'abord un utopiste, au meilleur sens du mot, rêvant de changer la société, avec des projets qui pouvaient d'abord paraitre complètement fous - mais il les réalisait. Comme il va nous manquer!

Bibliographie des travaux de René Berchoud : Le patois de Néronde, mémoire de DEA, 1985 ; Le Labyrinthe Inès de Castro, dans $G$ comme Hugo, textes réunis par Antoine Court et Roger Bellet, Saint-Étienne, Publications de l'Université, 1987, pp. 59-82 ; La Révolution à Néronde et dans les montagnes du matin, 2 volumes, Feurs, Claude Bussy éditions, 1989 ; " La Terreur à Saint-Just-la-Pendue ", dans La Révolution en Forez, Actes du colloque du 7 octobre 1989, Montbrison, Comité montbrisonnais du Bicentenaire de la Révolution française, 1989, pp. 67-73; Javogues et son image dans la mémoire collective de la région de Feurs, Feurs, collège du Palais, br., 1989, 20 p.; La Révolution à Feurs, documents des Archives municipales, Feurs, collège du Palais, 1989, 193 p. ; Les canettes de Jérôme Roquet, par Louis-Étienne Blanc, [texte en dialecte lyonnais], présentation, textes choisis et commentaires de René Berchoud, Claude et Danièle Latta, Néronde, ADN, 1997, 117 p. ; " Marguerite Gonon et Néronde ", dans Marguerite Gonon (1914-1996) [ouv. coll.], Montbrison, Village de Forez, 1997, pp. 38-40.

\section{NOTES}

${ }^{1}$. Sur Fernand Rude, voir notre étude biographique de Fernand Rude et la bibliographie de ses œuvres dans le $\mathrm{n}^{\circ} 7$ (1991) de notre revue, 1848-Révolutions et mutations du XIXe siècle, pp. 93-100, ainsi que l'article que lui consacre - sous la plume de Maurice Moissonnier - le "Maîtron", DBMOF, tome 41, 1992, pp. 21-22. 